\title{
TELEMEDICINE AND ONLINE PLATFORMS AS AN OPPORTUNITY TO OPTIMISE QUALITATIVE DATA COLLECTION, EXPLORE AND UNDERSTAND DISEASE PATHWAYS IN A NOVEL PANDEMIC LIKE COVID-19
}

\author{
Janet Michel RN, BACUR, MPH, PHD, Wolf E Hautz MD, MME, Thomas C Sauter MD, MME
}

Department of Emergency Medicine, Inselspital, Bern University Hospital, University of Bern, Switzerland

\begin{abstract}
This article explores digital technology used in the health sector today. There are several ways digital technology is being used for quantitative study aims during the current COVID-19 Pandemic e.g. online symptom checkers. The knowledge gap in COVID-19 as in all novel conditions, consist of both quantitative and qualitative attributes. Digital tool use is prevalent during the COVID-19 Pandemic but most of the data being collected is quantitative in nature. We therefore recommend taking advantage of this telemedicine era and explore optimisation of qualitative data collection, for the purpose of gaining a better understanding of the phenomenon.

Keywords: COVID-19; novel pandemics; telemedicine; mixed study designs; qualitative research

Michel J, Hautz WE, Sauter TC. JISfTeH 2020;8:e9(1-4).

Crossref

DOI: https://doi.org/10.29086/JISfTeH.8.e9

Copyright:@ The Authors 2020

Open access, published under Creative Commons Attribution 4.0 BY International Licence
\end{abstract}

\section{Introduction}

Digital technology is being used in many forms in the health sector today. ${ }^{1}$ Examples include enabling people to manage their health more effectively through monitoring of vital signs, use of dispensers to promote medication adherence, techniques to diagnose diseases better, to delivering health services in the form of telemedicine. ${ }^{1,2}$ Remote care and mobile health have been proven to increase patient safety through among others things, electronic sensors that track activity, measure vital signs and assist staff in monitoring patients at risk of falls. ${ }^{2}$

Digital technology has also improved the way data are gathered, analysed, managed and exchanged in all health sectors, improving health information systems from community, district, provincial, national to global levels. ${ }^{2}$ Accuracy of data collection, timelines and reporting, disease monitoring and surveillance, have all greatly improved as a result. ${ }^{1}$ In low-income settings, innovative digital technologies such as handheld ultrasound and pulse oximeters have resulted in effective diagnosis and improved outcomes. $^{2}$

There are several ways digital technology is being used for quantitative studies during this current Pandemic:

- Artificial Intelligence (AI) to detect and anticipate the spread of COVID-193

- Online symptom checkers ${ }^{4}$
- Interactive maps and online dashboards to visualise and track reported cases on a daily basis e.g. WHO COVID19 dashboard $^{5}$

- New technology for faster detection and diagnosis of COVID-19 is under production e.g. portable lab-on chip detection $\mathrm{kit}^{3}$

- Genome sequencing to find potential vaccines ${ }^{3}$

- Robots to care for the infected in the context of high mortality among health workers ${ }^{3}$

An educated, aware and engaged public is one of the health system goals and there are many patient communities and online platforms that have been set up during this COVID19 pandemic context with the ntention to achieve this goal. ${ }^{6,7}$ Known COVID-19 symptoms include, sore throat, fever, breathing difficulties, rapid heart rate, kidney failure, chest pain, chills, shortness of breath, cough, rhinitis, and temporary loss of smell and taste. ${ }^{3}$ Gastrointestinal symptoms like vomiting and diarrhoea have been added to the list recently. ${ }^{4}$ Little is known about the subjective impact of these or other yet to be discovered symptoms, on patients self-caring at home and in ambulatory settings.

Telehealth is allowing patients to stay at home and consult healthcare workers in their home comforts. ${ }^{8}$ This reduces the burden on the health system and prevents infected patients from travelling and spreading infection. ${ }^{9}$ At the same time, many patients are being discharged into the community as 
early as possible to further self-quarantine, while alleviating pressure on hospitals. ${ }^{10}$ Digital technology hence increases, self-care, access to healthcare services at a distance ${ }^{1}$ and in some instances even opportunities to conduct research. ${ }^{11}$

\section{Qualitative data collection - an opportunity}

The knowledge gap in COVID-19 as in novel conditions in general, consists of both quantitative and qualitative attributes. As revealed above, the use of digital tools is prevalent during the COVID-19 Pandemic but most of the data being collected are quantitative in nature. ${ }^{7}$ 'Mixed method' is a research approach that systematically integrates collection, analysis and integration of both quantitative and qualitative data at some stage for the purpose of gaining a better understanding of the phenomenon. ${ }^{12}$ The rationale for mixing both kinds of data is based on the fact that neither quantitative nor qualitative methods alone are sufficient by themselves to capture trends and details of a phenomenon. When used in combination quantitative (understanding) and qualitative (confirming or testing) methods complement each other taking advantage of the strengths of each. ${ }^{13}$

The understanding of disease and transmission patterns e.g. risk factors, severity and clinical features of a novel condition like COVID-19 is limited at the beginning. ${ }^{14}$ The feelings, meaning and experiences of COVID-19 patients in dealing with loneliness, fear and self-care can better be understood qualitatively. Telemedicine is being used to keep people in touch during this social distancing and lockdown period, even offering patients the ability to talk to therapists when scared, anxious or depressed. Many patients have used video calls to keep in touch with loved ones. ${ }^{3,9}$ How are those infected with COVID-19, sick and quarantined at home feeling and experiencing the disease? Who can they talk to when anxious or not sure of symptoms just to get reassurance? ${ }^{1,15}$

\section{Proposal to tap into the largest COVID-19 patient group - those with mild symptoms self- caring at home}

The authors propose that future research both tap into the largest COVID-19 patient group available (those with mild symptoms self-caring at home) and better leverage collection and analysis of qualitative data. The COVID-19 disease pathway, progression, signs and symptoms are slowly being understood, but more data is needed to get a holistic picture of this condition. In the COVID-19 context, many patients are encouraged to stay at home, self-isolate and self-care. Patients consult a physician and report symptoms they are experiencing on the day of consultation. Symptoms and other disease-related signs they may experience between diagnosis and recovery are rarely captured. As the majority of patients will self-care at home, to prevent overloading of the health system, we propose to take advantage of this data source (those who are sick and self-care at home) and collect additional qualitative data using digital technology; videos, SMS, voice notes, e-diaries. Patients would report their symptoms daily with prompts sent out if the day's entry was not recorded (to reduce recall bias). The data would be fed into a live database updated daily according to day postdiagnosis, e.g. day 1, 2, 3 etc. and then coded for themes.

1. Patients could be encouraged to record symptoms and experiences daily via either video, voice notes, SMS daily journaling ( e-diary) explaining how they experience the illness, signs, symptoms, fear, anxiety etc. from diagnosis to recovery. Recording their feelings and disease experience would help develop a clearer picture of the condition and how it manifests over time. ${ }^{1}$ In support of our proposal, recent research revealed that teenagers are twice as likely to become infected with COVID-19 than younger children ${ }^{16}$ and that it is important for schools and communities to monitor multiple indicators of COVID19 among school-aged children as schools reopen. ${ }^{16}$ To contain COVID-19, online forward triage tools (OFTT) ${ }^{2}$ could be used in these settings to screen and diagnose children who exhibit symptoms as well as give advice to those isolating at home. These OFTTs could collect qualitative data like symptoms experienced and if other contacts are exhibiting symptoms. Daily check-in calls or SMS could aid in collecting qualitative data on the presentation of COVID-19, characteristics and health outcomes in this age group as little is yet known. ${ }^{1,16}$ There are currently hundreds of unplanned and undocumented natural experiments taking place in schools across America, but as the data are not being systematically gathered, one cannot learn from them and an opportunity is lost, ${ }^{17}$ hence our proposals.

2. Randomised controlled trials (RCTs) could be designed incorporating different data collection techniques video, voice note SMS and e-diaries. At the end of a quarantine or after recovery, an assessment of symptoms, feelings and experiences captured using the three methods (comparing and contrasting similarities and differences in feelings of anxiety, worry or neglect from medical teams in the three groups, comparing and contrasting if the methods captured known, listed symptoms and identifying new, yet unknown symptoms recorded and bring these to light). Methods that best serve the patient e.g. if video calls are identified as more effective in allaying fear and anxiety as compared to voice notes and SMS etc could be an additional contribution to the body of knowledge. The viability of telephone interviews in collecting rich narrative data has been proven. ${ }^{18}$ Short message services have been used to deliver health information and have been proven effective in reducing maternal and child mortality. ${ }^{19}$ We propose an adapted approach where the SMS is sent from the patient to health care provider, daily to report symptoms, progress and outcomes. In the COVID-19 context, social-distancing is 
recommended and it is responsible to do so. Qualitative data, however, is often collected through face to face interviews or focus group discussions, a questionable, difficult and possibly unethical thing to do during the COVID-19 Pandemic. A recent study has revealed that video-conferencing software like Zoom, effectively supports qualitative data collection in research, even extending to diverse and geographically dispersed populations. $^{20}$

\section{Moving forward}

As schools and companies reopen, OFTTs are being recommended for screening students and employees before they enter the school or work place. ${ }^{14,21,22}$ Clinicians, scientists, policy makers and practitioners are all keen to acquire more knowledge on COVID-19 infection, including its clinical presentation, the course of associated diseases, how secondary infections manifest and infection rates among different population and age groups as well as different settings. ${ }^{3}$ Most of the data currently being collected is quantitative in nature, hence we propose to incorporate prospective qualitative data collection methods ${ }^{20}$ like Zoom interviews, ${ }^{20}$ telephone, ${ }^{11}$ patient diaries ${ }^{22}$ and short message service (SMS) so as to get in-depth, rich knowledge and narratives that facilitate a rounded understanding of the novel infection, COVID -19.

\section{Corresponding author: \\ Janet Michel \\ Department of Emergency Medicine \\ Inselspital \\ Bern University Hospital \\ University of Bern \\ Switzerland. \\ Email:janetmichel71@gmail.com}

Conflict of interest. The authors declare no conflicts of interest.

\section{References}

1. Whitelaw S, Mamas MA, Topol E, Spall HGCV. Applications of digital technology in COVID-19 pandemic planning and response. Lancet Digit Health 2020;2(8):e435-e440. DOI: https://doi.org/10.1016/S2589-7500(20)30142-4

2. WHO. Digital technologies: shaping the future of primary health care. (2018). Available at: https://www.who.int/docs/default-source/primaryhealth-care-conference/digitaltechnologies.pdf? sfvrsn $=3 \mathrm{efc} 47 \mathrm{e} 0 \_2$ accessed 30 September 2020.
3. The Medical Futurist. How Digital Health Technology Can Help Manage The Coronavirus Outbreak. (2020). Available at: https://medicalfuturist.com/how-digitalhealth-technology-can-help-manage-the-coronavirusoutbreak accessed 15 April 2020.

4. Chambers D, Cantrell AJ, Johnson M, et al. Digital and online symptom checkers and health assessment/triage services for urgent health problems: systematic review. BMJ Open 2019;9(8):e027743. DOI: http://dx.doi.org/10.1136/bmjopen-2018-027743

5. World Health Organization. WHO Coronavirus Disease (COVID-19) Dashboard. (2020). Available at: https://covid19.who.int/ accessed 22 April 2020.

6. eNotfallmedizin. (2020) Available at: https://www.enotfallmedizin.ch/ accessed 22 April 2020.

7. SMASS. Covid-Guide. (2020). Available at: https://covidguide.smass.ch/\#/pathfinder/chatbot accessed 30 September 2020.

8. Maeder A, Bidargaddi N, Williams P. Contextualising digital health contributions to fighting the COVID-19 Pandemic. J Int Soc Telemed EHealth 2020;8:e3(1-5). DOI:10.29086/JISfTeH.8.e3

9. Siwicki W. Healthcare IT News. Telemedicine during COVID-19: Benefits, limitations, burdens, adaptation. (2020). Available at:

https://www.healthcareitnews.com/news/telemedicineduring-covid-19-benefits-limitations-burdensadaptation accessed 22 March 2020.

10. Crowe S, Brown K, Tregay J, et al. Combining qualitative and quantitative operational research methods to inform quality improvement in pathways that span multiple settings. BMJ Qual Saf 2017;26(8):641-652. doi:10.1136/bmjqs-2016-005636

11. Holt A. Using the telephone for narrative interviewing: a research note. Qual Res 2010;10(1):113-121. DOI: https://doi.org/10.1177/1468794109348686

12. Creswell JW, Guetterman TC. Educational Research: Planning, Conducting, and Evaluating Quantitative and Qualitative Research, 6th Edition. Upper Saddle River, New Jersey, Pearson, 2019. Available at: http:/content/one-dot-com/one-dot-com/us/en/highereducation/program.html accessed March 23, 2020.

13. Morse JM. Determining sample size. Qual Health Res 2000;10(1):3-5. DOI:10.1177/104973200129118183

14. Centers For Disease Control And Prevention. Coronavirus Disease 2019 (COVID-19). (2020). Available at: https://www.cdc.gov/coronavirus/2019ncov/hcp/index.html accessed 22 March 2020.

15. Gilchrist K. Psychology experts share their tips for safeguarding your mental health during quarantine. CNBC. (2020). Available at: https://www.cnbc.com/2020/03/20/coronavirus-tipsfor-protecting-your-mental-health-duringquarantine.html accessed 8 April 2020. 
16. Leeb RT, Price S, Sliwa S, et al. COVID-19 Trends Among School-Aged Children - United States, March 1-September 19, 2020. MMWR Morb Mortal Wkly Rep 2020;69(39):1410-1415.

DOI: $10.15585 / \mathrm{mmwr} . \mathrm{mm} 6939 \mathrm{e} 2$

17. Mandavilli A. New York Times. The Coronavirus Mostly Spares Younger Children. Teens Aren't So Lucky. (2020) Available at:

https://www.nytimes.com/2020/09/28/health/coronavir us-children.html accessed September 29, 2020.

18. Drabble L, Trocki KF, Salcedo B, Walker PC, Korcha RA. Conducting qualitative interviews by telephone: Lessons learned from a study of alcohol use among sexual minority and heterosexual women. Qual Soc Work QSW Res Pract 2016;15(1):118-133. doi: $10.1177 / 1473325015585613$

19. Haryanti RH, Hastuti TK, Hanim D. Short Message Service (SMS) Bunda: Innovation policy to reduce maternal mortality in Brebes. KnE Soc Sci 2017;2(4):69-75. DOI:10.18502/kss.v2i4.869

20. Gray L, Wong-Wylie G, Rempel G, Cook K. Expanding qualitative research interviewing strategies: Zoom video communications. Qual Rep 2020;25(5):1292-1301.

21. Healthcare IT News. Back to work during COVID-19: A symptom checker can make for a safer return. (2020). Available at: https://www.healthcareitnews.com/news/emea/backwork-during-covid-19-symptom-checker-can-makesafer-return accessed 30 September 2020.

22. Bitran H, Gabarra J. The Official Microsoft Blog. Delivering information and eliminating bottlenecks with CDC's COVID-19 assessment bot. (2020). Available at: https://blogs.microsoft.com/blog/2020/03/20/deliverin g-information-and-eliminating-bottlenecks-with-cdescovid-19-assessment-bot/ accessed 30 September 2020. 